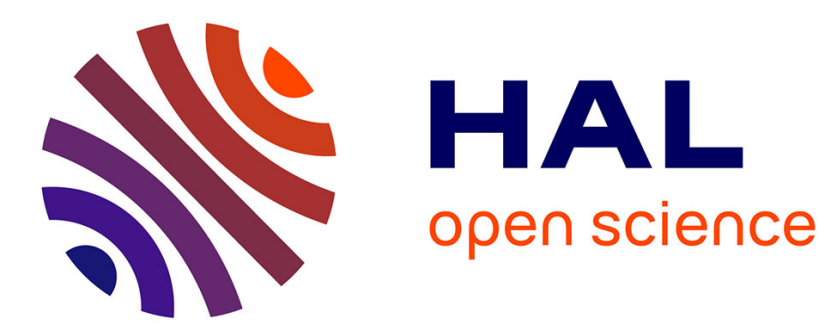

\title{
Sense2Health: A Quantified Self Application for Monitoring Personal Exposure to Environmental Pollution
}

Sara Hachem, Georgios Mathioudakis, Animesh Pathak, Valérie Issarny, Rajiv Bhatia

\section{To cite this version:}

Sara Hachem, Georgios Mathioudakis, Animesh Pathak, Valérie Issarny, Rajiv Bhatia. Sense2Health: A Quantified Self Application for Monitoring Personal Exposure to Environmental Pollution. SENSORNETS 2015, Feb 2015, Angers, France. hal-01102275

\section{HAL Id: hal-01102275 \\ https://hal.inria.fr/hal-01102275}

Submitted on 12 Jan 2015

HAL is a multi-disciplinary open access archive for the deposit and dissemination of scientific research documents, whether they are published or not. The documents may come from teaching and research institutions in France or abroad, or from public or private research centers.
L'archive ouverte pluridisciplinaire HAL, est destinée au dépôt et à la diffusion de documents scientifiques de niveau recherche, publiés ou non, émanant des établissements d'enseignement et de recherche français ou étrangers, des laboratoires publics ou privés. 


\title{
Sense2Health: A Quantified Self Application for Monitoring Personal Exposure to Environmental Pollution
}

\author{
Sara Hachem ${ }^{1}$, Georgios Mathioudakis ${ }^{2}$, Animesh Pathak ${ }^{2}$, Valérie Issarny ${ }^{1}$, and Rajiv Bhatia ${ }^{3}$ \\ ${ }^{1}$ Inria@Silicon Valley, Berkeley, USA \\ ${ }^{2}$ Inria Paris-Rocquencourt, Le Chesnay, France \\ ${ }^{3}$ The Civic Engine, Berkeley, USA \\ \{sara.hachem, georgios.mathioudakis, animesh.pathak, valerie.issarny\}@inria.fr, rajivxbhatia@gmail.com
}

\begin{abstract}
Keywords: mobile sensing, quantified self, environment monitoring, well-being, noise sensing.
Abstract: $\quad$ Sense2Health is a Quantified Self application that monitors personal exposure to environment pollution and assesses its heath-related risks. The novelty of the application is that it requires little to no active involvement by users and unlike existing applications, it correlates the individual's well-being to their environment as opposed to their physical activity alone. Consequently, when health and environment data are acquired, our application enables users to better identify behavior changes towards enhancing their health by enhancing their environments. Furthermore, Sense2Health is an open platform for integrating existing domain-specific sensing applications (environmental and health monitoring) focused on decreasing required specialized development efforts. We present in this paper the design of Sense2Health in addition to a proof-of-concept implementation for a noise-monitoring use case. Afterwards, we assess its performance while integrating it with a dedicated open source noise sensing application.
\end{abstract}

\section{Introduction}

In Quantified Self applications, individuals are engaged in monitoring their well-being by tracking specific physical or biological information, such as heart rate, daily physical activity, or sleep quality through their mobile devices (Swan, 2013). There are various reasons for the ongoing success of such applications. Specifically, in this information-oriented era, in addition to decreasing health risk factors and generating highly valuable information (Fritz et al., 2014), tracking one's data is considered fun and becoming an accepted social activity. However, work remains to be done towards personalizing the feedback loop between the application and users towards behavior change for better health. Additionally, a common trend in such applications is the focus on personal physical factors alone, while lacking efforts in analyzing/tracking the effects of the surrounding environment itself on individuals' well-being with the feedback representing environmental risks on health.

In fact, environment pollution is a major issue in cities, especially since 7 out of 10 people are expected to be living in urban areas by 2050 within a drastically increasing number of megacities consist- ing of a population exceeding 10 million people. ${ }^{1}$ As such, the health of the city has a direct impact on the health of the citizen, for example through exposures to environmental agents such as air pollutants (Burnett et al., 2014; Brauer et al., 2012). ${ }^{2}$ Additionally, monitoring environmental pollution and increasing personal awareness on the matter - albeit mostly restricted to air monitoring with no assessment of the exposure repercussions - has been gaining momentum as illustrated by an increasing number of industrial efforts motivating users to perform the monitoring tasks (http: / / www. plumehq. com, http: // www . clarify.io).

The best approach to understanding, visualizing and assessing the aftereffect of direct exposure to - and conversely fighting against - an environmentally polluted surrounding is by constantly monitoring one's health (blood pressure, heart rate, etc.). A few years back, such a requirement would have been a dreadful, if not an impossible task. However, with

\footnotetext{
1 http://www.who.int/gho/urban_health/ situation_trends/urban_population_growth_ text/en

2 http://www.who.int/phe/health_topics/ outdoorair/databases/AAP_BoD_results_ March2014.pdf
} 
today's proliferation of biosensors, self monitoring is turning into a trivial task that simply requires the user to own a recent smartphone, smartwatch or smart bracelet. Yet, little to no effort has been provided towards integrating personal health data with urban data in order to assess well-being while inducing behavior change for a healthier environment, healthier community, and consequently a healthier life.

To assist users in tracking their well-being with respect to environmental pollution and understand its effect on their health, we provide an application that can leverage, altogether, biosensors and available domain-specific environment monitoring mobile applications that track a phenomenon of interest, such as noise pollution. The latter is a critical healthjeopardizing source of environmental pollution. Yet, it has been tackled mostly for city planning purposes with little effort to enable users to understand the effects of their own exposure to noise. Evidently, there are various sources of pollution that, to our day, cannot yet be measured with mobile sensors alone, such as chemical pollutants. However, given the fast pace of technical advancements and increasing number of sensors integrated in mobile devices, we consider this to not be an issue.

Our contribution in this paper is threefold:

1. Design Sense2Health as an environmental pollution-oriented Quantified Self application that requires little active involvement from end users.

2. Design Sense2Health to be a resource-efficient and development-efficient platform, i.e., decrease required coding efforts. The latter is ensured by leveraging existing efforts in the area of mobile sensing for environment and health monitoring and integrating domain specific sensing applications with ours.

3. Assessment of the performance of Sense2Health, through a noise monitoring proof-of-concept implementation and integration with a dedicated noise sensing application. Our goal is to better understand the effects of integrating various sensing applications on the device's resources and inform future design and implementation work.

The paper is structured as follows: Section 2 gives an overview of the literature. In Section 3, we present the architecture of Sense2Health, followed by the noise-specific implementation in Section 4. The performance evaluation of Sense2Health is presented in Section 5. Finally, in Section 6, we give a summary of our contributions and planned future work.

\section{Related Work}

Well-being applications are rather popular. They allow users to track their physical activity and fitness and share it along their social networks. However, they still suffer from various drawbacks. We present the drawbacks in this section as we review several existing well-being applications, followed by an overview of solutions for noise monitoring, which is our use case for environment-related well-being monitoring.

\subsection{Mobile Applications for Well-Being}

Well-being assessment applications are still growing in popularity, especially with the increasing awareness of the importance of health monitoring and the easier access to various types of sensors. However, this trend is still limited by two characteristics: the sensing is manual and/or relies on external devices that mainly provide heath-related data (Swan, 2013).

Among such devices, Fitbit (http://www. fitbit.com) is a bracelet that allows users to monitor their sleep habits and physical activity. DirectLife (http://www.directlife.com) is an activity program with an activity monitoring device that coaches the user towards creating a personal activity plan. Authors in (Seong et al., 2014) propose the use of smartwatches to collect data from external devices through peer to peer communication to enable users to track their physical activities. Authors in (Angelini et al., 2013) present a smart bracelet for elderly people to act as a personal assistant and monitory their health status, remind them to take their medications, etc. Balance (Denning et al., 2009) is a system that automatically detects the user's caloric expenditures via sensor data provided by a sensing unit on the user's hip. Users are required to manually input information on food intake through the mobile application. AndWellness (Hicks et al., 2010) is a personal data collection system that uses mobile phones to collect and analyze data. However, data is collected through surveys with context data and sensor data, mainly GPS location and physical activity inference. The Mobile Coach is a well-being application presented in (Ahtinen et al., 2009), which similar to above, also relies on manual user provided input. It generates training plans based on user provided personal goals and performed workouts, which are also input manually. Google Fit (http://developers.google.com/fit/) is a platform to integrate various health monitoring applications that leverage biosensors. Similar efforts are provided by Apple within the HealthKit tool (https: //developer.apple.com/healthkit/). Microsoft 
Health Vault (http://www.healthvault.com/fr/ en) service combines a cloud-based service with a device-hosted middleware to provide developers of fitness applications and devices the ability to upload all data to a central clearinghouse.

As illustrated by the applications above, in addition to being manual, the majority of the solutions focus on fitness and physical activity as the basis of well-being assessment without accounting for environment-related health nuisances.

\subsection{Noise Monitoring}

Unlike the above well-being applications which require, in many cases, active involvement of users, noise pollution monitoring constitutes an adequate example for automatic sensing where data is collected from microphones on users' devices without necessarily requiring their active involvement in the process. However, existing noise sensing applications are restricted to basic feedback that shows users noise values in Decibels (Bennett et al., 2010) or noise maps (Bennett et al., 2010; Rana et al., 2010; Maisonneuve et al., 2010) with basic graphs that plot noise values over time. For instance, NoiseSPY (Kanjo, 2010 ) is a mobile platform for urban noise monitoring and mapping that allows users to measure, annotate and localize noise pollution in a city through crowd sensing. The platform provides them with access to maps and a noise plot graph for a certain time duration or along a trip. NoiseTube (Maisonneuve et al., 2010) is a participatory noise monitoring solution focusing on real-time exposure to noise experienced by citizens. Noise is sensed through users' smartphones and complemented with user generated contextual data (location, time, and noise source), along with machine-based automatic classifiers for e.g., time, location, weather and activity identification. The output is a noise map and a log of personal exposure to noise with a graph that plots the value of noise throughout the day. While such information is important, it is not sufficient as users need a qualification rather than quantification of the noise pollution along with better vizualization to be able to form an informed opinion.

Furthermore, focus in existing solutions e.g., (Rana et al., 2010; D'Hondt et al., 2013; Bennett et al., 2010) is mostly on noise measurement itself, especially in terms of sensor calibration or measurement/maps accuracy and quality while work remains to be done in order to properly represent the results to users so as to increase their awareness of their surrounding environment pollution, which can be a major harming factor to their well-being.

\section{Sense2Health Application Design}

As stated earlier, the main purpose of the Sense2Health application is to enable users to track, analyze and correlate well-being states with personal exposure levels to an environmental phenomenon, e.g., noise, and do so with the least active involvement possible through automatic sensing (and bio-sensing). Our goal is not to deliver yet another application that performs the actual sensing. In more detail, our design rational was to provide a platform that communicates with and integrates sensing applications and their measurements, and also enables users to personalize the application according to their own habits (to better assess their well-being) and grants them access to processed and visual data, while simultaneously ensuring resource efficiency. All those requirements are satisfied by the various Sense2Health components depicted in Figure 1 and presented, in more detail, below. It should be noted that we distinguish between two types of data: raw data, which is the measurement provided by the sensing application and consists of a numerical value that quantifies the state of the feature of interest and aggregated data which is a (set of) raw measurement(s) that underwent some processing within the Sense2Health application. Data related to the user's health is referred to as health data and data related to the environment is referred to as environmental data.

The Profile Manager allows users to specify their preferences and setup their profile. The preferences include information on whether or not the user desires to enable automatic sensing and enable power saving options to dynamically modify sensing properties according to available resources. Creating a profile is essential, as it includes information on users' daily schedules, such as sleeping hours and working hours which enables the application to better assess the impact of the phenomenon on their well-being. For instance, an average noise exposure of $60 \mathrm{db}$ may be acceptable during the daytime, however, the same level can disturb sleep in a portion of the population at night (Hurtley, 2009).

The Measurement Manager is the component that assists with the integration of various sensing applications, as it directly interacts with them to acquire their measurements based on the sensing settings provided by the Resource Manager and the user preferences in the Profile Manager. Sensing applications generate environmental data by leveraging ambient sensors (e.g., NoiseDroid ${ }^{3}$ monitors noise using microphones, Plume monitors air quality using dedicated sensors,

\footnotetext{
3 https://wiki.52north.org/bin/view/ SensorWeb/OpenNoiseMap
} 


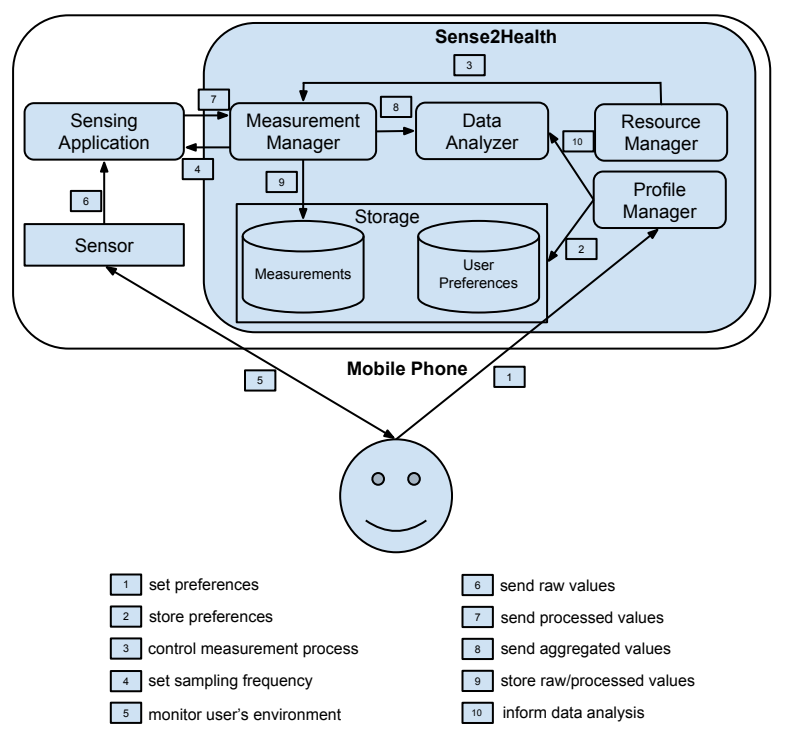

Figure 1: The Sense2Health architecture.

etc. ) and health data by leveraging biosensors (e.g., FitBit). The component manages the automatic sensing process and enables users to request manual sensing. It periodically forwards appropriate data from bio and ambient sensors to the Data Analyzer.

The Data Analyzer handles the processing and aggregation of health and urban data to generate exposure and health risk assessment graphs, personalized based on users' profiles to dynamically determine whether or not the phenomenon is considered to be harmful. The graphs should enable users to visualize their hourly, daily and monthly exposure to the phenomenon, and view historical data spanning previous days and months. The component also provides statistics on the frequency of potentially harmful noise exposures. This component can be perceived as the component that closes the feedback loop by providing the relevant information to the user. It is worth mentioning that there are various medical studies and research efforts that we can leverage when assessing and correlating environmental pollution and health risks (Miedema, 2007). ${ }^{4}$

The Resource Manager ensures the efficiency of the application by tracking power and resource consumption in order to modify the sensing frequency and sampling duration dynamically. In particular, the sampling should stop if the battery's resources are low while its frequency is increased if the phone is being charged. There are various solutions that can be ex-

\footnotetext{
${ }^{4}$ http: //www. who.int/phe/health_topics/ outdoorair/databases/AAP_BoD_results_ March2014.pdf
}

ploited for optimized resource consumption, such as the approach proposed in (Lane et al., 2013) to piggyback on running applications in order to decrease sensory data collection overhead or the solution proposed in (Priyantha et al., 2011) to save energy by exploiting low-power sensing processors.

The Storage component holds all measurements (raw and aggregated values) and user profile information along with their preferences. We do not require data to be stored on the phone indefinitely. An option should be available to allow users to specify whether or not they want the raw data to be stored. However, to increase the application's efficiency when displaying the graphs, all aggregated data will be stored (so as to not be obliged to recompute all the displayed values at every request) until the observation history is cleared by the user. Additionally, periodic backups will take place by sending data to a remote store.

\section{Use case: Sense2Health for Noise Monitoring}

Sense2Health is a prototype mobile application for the Android platform through which users can monitor their health and their exposure to environmental factors by leveraging the sensing functionality provided by their mobile devices. Sense2Health introduces a Sensing API that enables the integration of several domain-specific sensing applications. In addition, it offers an intuitive user interface to facilitate the visual representation of the data and aggregated 
information. The interface was designed based on the guidelines in (Gong and Tarasewich, 2004). Data are stored locally on the device by the Storage component and can be transmitted to a backup server for more permanent storage. Sense2Health acts as a clearinghouse of data collected by other domain-specific applications. Its functionality can be accessed through the Java methods available in a companion . jar file that other applications can import and use. Internally, this library is a wrapper that uses standard Android inter-process communication through AIDL to connect to the Sense2Health application installed on the phone, which then stores the data.

\subsection{The Sensing API}

To provide an appropriate platform for hosting already existing sensing applications, a set of interfaces and methods, presented below, is introduced to define the Sensing API. The latter is designed based on the APIs of available open-source sensing applications such as NoiseDroid.

- void monitoringSetUp() - Includes tasks required for running the sensing application.

- void monitoringShutDown() - Includes tasks required for shutting down the sensing application.

- MonitoringValue conceptCapture() - Is used to perform a single sensing task and returns an object representing the state of the phenomenon to monitor. The sampling duration is parameterized.

- void addValueChangedListener() - Attaches listeners to track sensing events that may occur.

- void removeValueChangedListener() - Removes the specified listener.

- boolean isCapturing() - Checks if there is an ongoing sensing task.

The data requests to the sensing tasks are performed by the MeasurementsManager, an orchestrator class, which also transfers the data to the local storage component and a backup server. Additionally, the class enables Sense2Health to automatically request sensing tasks, even while running in the background, by leveraging Android built-in functionalities.

However, resources on mobile devices are rather limited. Therefore, Sense2Health should be modest on resource consumption (CPU \& Memory), thus leading to better satisfied users. The ResourceManager tracks the available resources and monitors their changes through a set of defined methods such as, getCPUUsage(). After- wards, taking into account all available information, the sensing processes are adjusted accordingly through adjustAutomaticSensing (), which is triggered asynchronously. Note that if the battery percentage is lower than $30 \%$ and the device is not charging, all sensing tasks are suspended.

The noise sensing case The noise sensing case is most suitable for evaluating Sense2Health since, on the one hand, the needed sensor (i.e., microphone) is available in any mobile device, and on the other hand, there is a plethora of Android applications that perform sound monitoring.

We leverage the NoiseDroid application, which is an open source project, used for collecting information on noise pollution. Its straightforward implementation and high accuracy on sound capturing made it the ideal candidate for integration with Sense2Health. In fact, the components responsible for the sensing task were integrated seamlessly with Sense2Health using the interfaces presented above. The noisespecific monitoring is performed by implementing the sensing methods as follows.

- monitoringSetUp(): Loads the audio settings (e.g., rate, channel, format of audio to be recorded) and initializes any functionalities needed by the recorder.

- monitoringShutDown(): Stops recording and releases the native AudioRecord resources.

- addValueChangedListener(): Informs listeners of value updates. Consequently, during a recording, NoiseDroid can report new sound values, enabling activities to track and display them.

- removeValueChangedListener(): Removes listeners.

- iscapturing (): Checks if the recorder has an ongoing sensing task.

Prior to further aggregation and storage, the values originating from the sensing tasks are structured internally using a Measurement class. A Measurement instance holds the specifics of the sensing task, which include the $\mathrm{min} / \mathrm{max} /$ average sound level (in Decibels) along with metadata provided by Sense2Health to describe the context of each measurement (e.g., location, date \& time, sensing duration, etc.).

\subsection{Data aggregation}

The goal of data aggregation is to extract valuable insights by processing environmental and health data and closing the feedback loop by returning the information to the user. Users will have the means to 


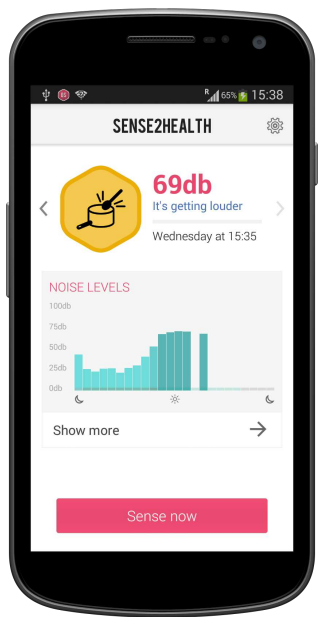

(a) The Home activity

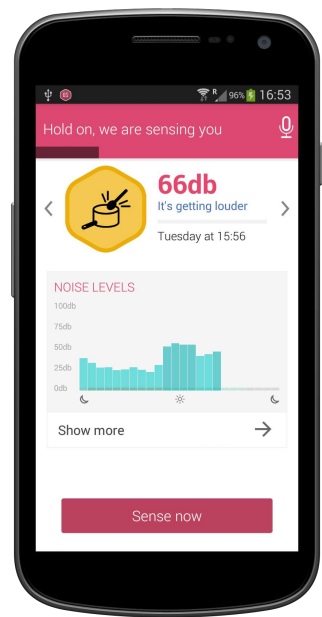

(b) Home during sensing

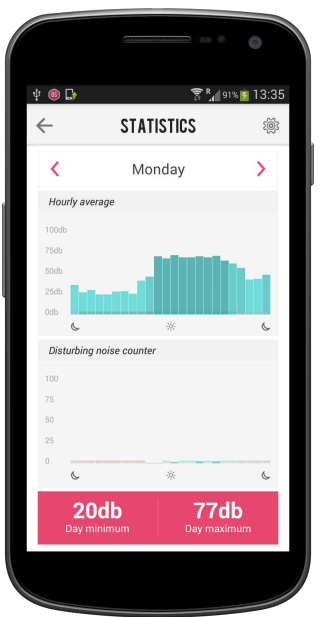

(c) The Graphs activity

Figure 2: GUI of the Sense2Health mobile application

track their physical state and exposure to urban phenomena (noise) over time and identify situations that affect their well-being and update their behavior towards a better environment and healthier community accordingly. In this first implementation, we focus on environmental data only. We introduce two forms of aggregated information. The first uses a simple average mechanism to showcase exposure over short and long time periods (hour, day, month, etc.) informing the users of their subjections to an environmental factor over time. The task is carried out by the calculateAverages() method, which takes as input the measurement (i.e., noise values), start/end date and the calculation unit.

The second form of aggregated information, presented as a counter, Disturbing Situation Counter (DSC), is used to keep track of those unpleasant moments where the sensing values reveal significant, potentially harmful, exposure. For instance, in the noise sensing example, the DSC identifies such incidents using a set of predefined thresholds (expressed in Decibels and extracted from medical studies by WHO on noise disturbance ${ }^{5}$ ), which are employed to classify noise levels (quiet, normal, loud, extreme) and then, disturbance. To generate the results for the counter, the generateDSC () method is used. The method takes as input the measurement (i.e., noise values), the start/end date and the unit (e.g., hour, day, etc.). All aggregated data are presented through interactive graphs (Figure 2(c)).

\footnotetext{
5 http://whqlibdoc.who.int/hq/1999/a68672. pdf
}

\subsection{Data management - Local storage}

Sensing applications often produce large amounts of data that need to be tracked, thus leading to an information-rich data store. Sense2Health addresses such need by introducing a DatastoreManager class that wraps an SQLite (http://www.sqlite.org/) database and provides methods to create/update/delete entries on it. The use of SQLite not only ensures high performance but also provides means for efficient information retrieval.

To prevent the database from occupying considerable storage space on a mobile device, we defined a policy to filter the data to be stored and the data to be removed. According to this policy, raw data are safe to be deleted after one month of their creation date, while aggregated data produced by the Data Analyzer are stored locally up to one year. Furthermore, the user can request, at any time, the removal of all the data, raw and aggregated, produced by the application. In particular, the DatastoreManager includes, among others, the following methods: insertMeasurement() - used to add a new measurement instance, updateMeasurement () - used to update attributes in a stored measurement and periodicCleanUp () - executed once a day to remove raw data from the database.

\subsection{Information visualisation}

To ensure a user-friendly experience when interacting with Sense2Health, we introduce a balanced user interface defined by involving users and preventive medicine practitioners in the design process. The interface (Figures 2(a), 2(b)) comprises 3 main parts: (i) 
The Measurements Slider including the most recent measurements (up to 24-hours old), (ii) The hourly average graph of the current day and the (iii) Sense Now button for triggering an immediate sensing task. Each item contained in the Measurements Slider includes a respective icon to visualise the sound level classification and a short hint providing a quick way for the user to understand the level of exposure without having to interpret the domain-specific metrics (e.g., Decibels).

The Statistics and Graphs screen (Figure 2(c)) can be accessed through the Show more button in the Home screen. As described in Section 4.2, two categories of graphs are included to showcase the exposure of the user to a phenomenon: The (hourly and daily) averages levels and the (hourly and daily) Disturbing Noise Counter. The different colors on the bars of the graphs represent the respective levels of the monitored feature (i.e., noise).

\section{Resource Consumption}

To acquire a better understanding and clearer guidelines for the design of a platform such as Sense2Health, which has its own performance (in terms of phone resources) depending on that of integrated applications, we assess the resource consumption that results from running Sense2Health with integrated NoiseDroid, in terms of CPU resource consumption and memory usage. We evaluated the application performance on Samsung Galaxy S3 Android phones with 2GB RAM. The results are presented throughout this section. It is worth noting that there are two distinct runtime phases in our application: the idle phase where no sensing/processing is performed, and the active phase when the actual sampling and processing take place. The active phase has a duration of 5 seconds every 10 minutes. We compare our application's resource consumption to that required by BeWell (Lane et al., 2011), a highly cited resourceefficient well-being application. The application was evaluated on a Nexus One smartphone.

\subsection{CPU Usage Benchmarks}

We monitored CPU usage by Sense2Health, each second over a duration of 3 hours. The results are presented in the graph in Figure 3. The CPU usage varies along the idle and active phases. The application requires around $2 \%$ of CPU resources in the idle phase while, during sensing and processing, it requires at most $22 \%$. Consequently, during the active phase, our application leads to a $20 \%$ increase, at most, in CPU

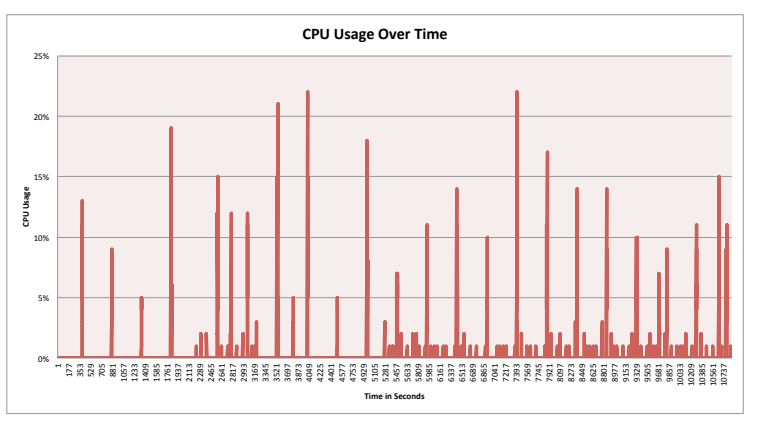

Figure 3: The percentage of CPU usage by Sense2Health.

usage. As compared to the BeWell application, which requires up to $31 \%$ of CPU resources and an MP3 player requiring up to $16 \%$ of the resources (Lane et al., 2011), we consider ours to have an acceptable CPU usage.

\subsection{Memory Usage Benchmarks}

We inspected the memory used by the Sense2Health process (Figure 4) by leveraging the $\mathrm{ADB}^{6}$ (Android Debug Bridge) tool, which reports the several types of memory allocation, namely, Proportional Set Size (PSS) and Private Dirty (PD).

The PSS is a measurement of the application's RAM usage, which accounts for sharing pages across processes. It is a good measure for the actual RAM weight of a process and for comparison against that of other processes. For Sense2Health the PSS is stable around $11.5 \mathrm{MB}$ with small variations due to garbage collection. The PD is the memory used by the process of interest alone (i.e. the Sense2Health process). This is the bulk of the RAM that the system can reclaim when the application's process is destroyed. For Sense2Health, the PD is stable around 9MB with variations, again due to garbage collection. As compared to BeWell, which requires up to $16 \mathrm{MB}$ and the MP3 player, which requires up to $26 \mathrm{MB}$, we consider the application's memory consumption to be low.

\section{Conclusion \& Future Work}

We presented in this paper Sense2Health, an application that enables individuals to monitor environmental data and assess its influence on their wellbeing in order to modify their behavior for a better, healthier environment and community. Sense2Health is designed as an open platform to enable seamless

\footnotetext{
${ }^{6} \mathrm{http}: / /$ developer.android.com/tools/help/adb.html
} 


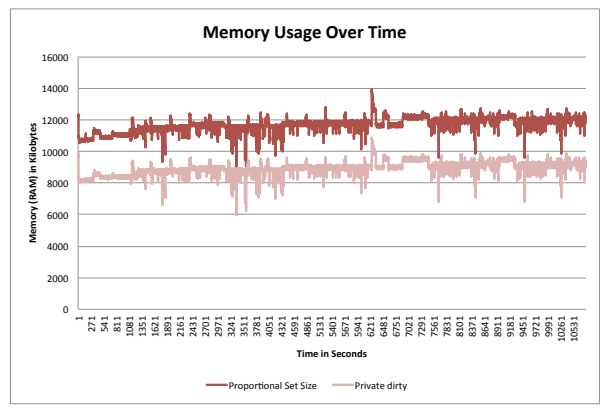

Figure 4: The Memory usage by Sense2Health.

integration of available urban/health monitoring solutions by leveraging ambient and biosensors. We presented the conceptual design of the platform and the companion application, followed by a description of a proof-of-concept implementation with noise monitoring. We also evaluated the performance of the application in terms of resource consumption, to better inform future enhancements when integrating various sensing applications.

As part of our Future Work, we plan on integrating biosensors to acquire physical data and provide further information on the correlation between urban pollution and the disturbance and harm inflicted on the user's health. We also plan on investigating other pollution use cases, such as air quality and identify potential enhancements to our platform. Additionally, we intend on extending our backup server into a scalable cloud-based store that can handle an ultra large number of users, store large volumes of data and protect user's privacy. To that end, we are investigating complementary solutions, such as Microsoft HealthVault, which enables users to store and share health information. Last but not least, we intend to integrate various domain specific sensing applications to identity and address potential constraints when various applications are simultaneously running within our platform.

\section{REFERENCES}

Ahtinen, A., Mattila, E., Vaatanen, A., Hynninen, L., Salminen, J., Koskinen, E., and Laine, K. (2009). User experiences of mobile wellness applications in health promotion: User study of wellness diary, mobile coach and selfrelax. In Pervasive Computing Technologies for Healthcare, 2009. PervasiveHealth 2009. $3 r d$ International Conference on, pages 1-8. IEEE.

Angelini, L., Caon, M., Carrino, S., Bergeron, L., Nyffeler, N., Jean-Mairet, M., and Mugellini, E. (2013). Designing a desirable smart bracelet for older adults. In Proceedings of the 2013 ACM conference on Per- vasive and ubiquitous computing adjunct publication, pages 425-434. ACM

Bennett, G., King, E. A., Curn, J., Cahill, V., Bustamante, F., and Rice, H. J. (2010). Environmental noise mapping using measurements in transit. In International Conference on Noise and Vibration Engineering, pages 1795-1810.

Brauer, M., Amann, M., Burnett, R. T., Cohen, A., Dentener, F., Ezzati, M., Henderson, S. B., Krzyzanowski, M., Martin, R. V., Van Dingenen, R., et al. (2012). Exposure assessment for estimation of the global burden of disease attributable to outdoor air pollution. Environmental science \& technology, 46(2):652-660.

Burnett, R. T., Pope, C. A., Ezzati, M., Olives, C., Lim, S. S., Mehta, S., Shin, H. H., Singh, G., Hubbell, B., Brauer, M., et al. (2014). An integrated risk function for estimating the global burden of disease attributable to ambient fine particulate matter exposure.

Denning, T., Andrew, A., Chaudhri, R., Hartung, C., Lester, J., Borriello, G., and Duncan, G. (2009). Balance: towards a usable pervasive wellness application with accurate activity inference. In Proceedings of the 10th workshop on Mobile Computing Systems and Applications, page 5. ACM.

D'Hondt, E., Stevens, M., and Jacobs, A. (2013). Participatory noise mapping works! an evaluation of participatory sensing as an alternative to standard techniques for environmental monitoring. Pervasive and Mobile Computing, 9(5):681-694.

Fritz, T., Huang, E. M., Murphy, G. C., and Zimmermann, T. (2014). Persuasive technology in the real world: a study of long-term use of activity sensing devices for fitness. In Proceedings of the 32nd annual ACM conference on Human factors in computing systems, pages 487-496. ACM.

Gong, J. and Tarasewich, P. (2004). Guidelines for handheld mobile device interface design. In Proceedings of DSI 2004 Annual Meeting, pages 3751-3756. Citeseer.

Hicks, J., Ramanathan, N., Kim, D., Monibi, M., Selsky, J., Hansen, M., and Estrin, D. (2010). Andwellness: an open mobile system for activity and experience sampling. In Wireless Health 2010, pages 34-43. ACM.

Hurtley, C. (2009). Night noise guidelines for Europe. WHO Regional Office Europe.

Kanjo, E. (2010). Noisespy: A real-time mobile phone platform for urban noise monitoring and mapping. Mobile Networks and Applications, 15(4):562-574.

Lane, N. D., Chon, Y., Zhou, L., Zhang, Y., Li, F., Kim, D., Ding, G., Zhao, F., and Cha, H. (2013). Piggyback crowdsensing (pcs): energy efficient crowdsourcing of mobile sensor data by exploiting smartphone app opportunities. In Proceedings of the 11th ACM Conference on Embedded Networked Sensor Systems, page 7. ACM.

Lane, N. D., Mohammod, M., Lin, M., Yang, X., Lu, H., Ali, S., Doryab, A., Berke, E., Choudhury, T., and Campbell, A. (2011). Bewell: A smartphone application to monitor, model and promote wellbeing. In 5 th International ICST Conference on Pervasive Computing Technologies for Healthcare, pages 23-26. 
Maisonneuve, N., Stevens, M., and Ochab, B. (2010). Participatory noise pollution monitoring using mobile phones. Information Polity, 15(1):51-71.

Miedema, H. M. (2007). Annoyance caused by environmental noise: Elements for evidence-based noise policies. Journal of social issues, 63(1):41-57.

Priyantha, B., Lymberopoulos, D., and Liu, J. (2011). Littlerock: Enabling energy-efficient continuous sensing on mobile phones. Pervasive Computing, IEEE, 10(2):12-15.

Rana, R. K., Chou, C. T., Kanhere, S. S., Bulusu, N., and Hu, W. (2010). Ear-phone: an end-to-end participatory urban noise mapping system. In Proceedings of the 9th ACM/IEEE International Conference on Information Processing in Sensor Networks, pages 105116. ACM.

Seong, K. E., Lee, K. C., and Kang, S. J. (2014). Self M2M based wearable watch platform for collecting personal activity in real-time. In Big Data and Smart Computing (BIGCOMP), 2014 International Conference on, vol., no, volume 286, pages 15-17.

Swan, M. (2013). The quantified self: Fundamental disruption in big data science and biological discovery. Big Data, 1(2):85-99. 